\title{
Erratum to: High expression of myocyte enhancer factor 2C (MEF2C) is associated with adverse-risk features and poor outcome in pediatric acute myeloid leukemia: a report from the Children's Oncology Group
}

\author{
George S. Laszlo ${ }^{1}$, Todd A. Alonzo ${ }^{2,3}$, Chelsea J. Gudgeon ${ }^{1}$, Kimberly H. Harrington ${ }^{1}$, Alex Kentsis ${ }^{4,5,6}$, \\ Robert B. Gerbing ${ }^{3}$, Yi-Cheng Wang ${ }^{3}$, Rhonda E. Ries ${ }^{1}$, Susana C. Raimondi ${ }^{3,7}$, Betsy A. Hirsch ${ }^{3,8}$, Alan S. Gamis ${ }^{3,9}$, \\ Soheil Meshinchi $i^{1,3,10}$ and Roland B. Walter ${ }^{1,11,12^{*}}$
}

\section{Erratum}

The original article [1] contains an omission of information in the Financial support sub-section of the Declarations section.

The authors would like to acknowledge the Andrew McDonough B+ Foundation for financial support of coauthor Dr Yi-Cheng Wang throughout this study, and regret this inadvertent omission from the original publication.

\footnotetext{
Author details

${ }^{1}$ Clinical Research Division, Fred Hutchinson Cancer Research Center, 1100 Fairview Ave N, D2-190, Seattle, WA 98109-1024, USA. '2Department of Biostatistics, University of Southern California, Los Angeles, CA, USA.

${ }^{3}$ Children's Oncology Group, Monrovia, CA, USA. ${ }^{4}$ Molecular Pharmacology and Chemistry Program, Sloan Kettering Institute, New York, NY, USA.

${ }^{5}$ Department of Pediatrics, Memorial Sloan Kettering Cancer Center, New York, NY, USA. ${ }^{6}$ Weill Medical College of Cornell University, New York, NY, USA. ${ }^{7}$ Department of Pathology, St. Jude Children's Research Hospital, Memphis, TN, USA. ${ }^{8}$ Department of Laboratory Medicine and Pathology, University of Minnesota Cancer Center, Minneapolis, MN, USA. ${ }^{9}$ Division of Hematology-Oncology, Children's Mercy Hospitals and Clinics, Kansas City, MO, USA. ${ }^{10}$ Department of Pediatrics, University of Washington, Seattle, WA USA. "'Department of Medicine, Division of Hematology, University of Washington, Seattle, WA, USA. ${ }^{12}$ Department of Epidemiology, University of Washington, Seattle, WA, USA.
}

Received: 17 November 2016 Accepted: 28 November 2016 Published online: 30 November 2016

\section{Reference}

1. Laszlo GS, et al. High expression of myocyte enhancer factor $2 \mathrm{C}$ (MEF2C) is associated with adverse-risk features and poor outcome in pediatric acute myeloid leukemia: a report from the Children's Oncology Group. J Hematol Oncol. 2015;8:115. doi:10.1186/s13045-015-0215-4.

\footnotetext{
* Correspondence: rwalter@fredhutch.org

${ }^{1}$ Clinical Research Division, Fred Hutchinson Cancer Research Center, 1100

Fairview Ave N, D2-190, Seattle, WA 98109-1024, USA

${ }^{11}$ Department of Medicine, Division of Hematology, University of

Washington, Seattle, WA, USA

Full list of author information is available at the end of the article
} 